\title{
Musik für Maschinen?! - \\ Wo sich die Wissenschaft der Medien, des Computers und der Musik treffen und wie sie zusammenarbeiten könnten
}

Shintaro Miyazaki, Berlin

DOI: $10.25366 / 2020.105$

Zitation: Shintaro Miyazaki, „Musik für Maschinen?! - Wo sich die Wissenschaft der Medien, des Computers und der Musik treffen und wie sie zusammenarbeiten könnten", in: Brückenschläge zwischen Musikwissenschaft und Informatik. Theoretische und praktische Aspekte der Kooperation, in Verbindung mit der Fachgruppe Digitale Musikwissenschaft hrsg. von Stefanie Acquavella-Rauch, Andreas Münzmay und Joachim Veit (= Musikwissenschaft: Aktuelle Perspektiven. Bericht über die Jahrestagung der Gesellschaft für Musikforschung 2019 in Paderborn und Detmold, Bd. 3), Detmold, Musikwissenschaftliches Seminar der Universität Paderborn und der Hochschule für Musik Detmold, 2020, S. 173-178, DOI: 10.25366/2020.105 


\title{
Wo sich die Wissenschaft der Medien, des Computers und der Musik treffen und wie sie zusammenarbeiten könnten
}

\author{
SHINTARO MIYAZAKI, BERLIN
}

Während nicht nur die Notation und Aufführung eher klassischer, sondern auch elektroakustischer Musik mit Hilfe digitaler Medien erfolgt und Audiotechnologien generell längst digital operieren, scheint es schwieriger und auch zuweilen etwas unsinnig über diese Einzelbereiche hinausgehend Methoden und Ansätze der Musikwissenschaft generell und im Zuge eines reinen Fortschritt- und Innovationsglaubens zu "digitalisieren“. Diese Form der Annäherung von Informatik und Musikwissenschaft geschieht üblicherweise im Bereich der sogenannten Digital Humanities. Mein Beitrag argumentiert kritisch-historisch mit Fokus auf technische und diskursive Kontexte der zweiten Hälfte des 20. Jahrhunderts und aus dem Standpunkt einer dritten Fachdisziplin, nämlich der Medienwissenschaft und Medientheorie. Was ich hier skizzieren möchte, ist kein ausgiebig erprobtes Material. Es sind eher Ansätze und Beschreibungen möglicher Brückenschläge zwischen Informatik und Musikwissenschaft, die in Forschung und Lehre exploriert und geprüft werden müssten.

Einleitend und brückenbildend fungiert die Frage nach der Beschaffenheit des Digitalen, die nicht nur klangliche oder musikalische Implikationen beinhaltet. Sie wird hier zuerst mit dem Hinweis auf meine bereits abgeschlossene, historisch-medienarchäologischen Studie auditiver Wissenspraktiken im Kontext früher Großrechner der 1940er, 50er und 60er-Jahre angedeutet. Danach wird erläutert wie bzw. woran die Wissenschaft der Medien, der Computer und der Musik zusammenarbeiten könnten, wenn es eben um jene Frage nach der Beschaffenheit des Digitalen, manche nennen es Digitalität, ${ }^{1}$ und auch darüber hinaus ihre Sozialität geht. Hier orientiere ich mich an der Rhythmusanalyse des marxistischen Philosophen Henri Lefebvre $(1901-1991)^{2}$

\section{Der Klang der Digitalität}

Musik und Informatik spielen nicht nur während der Aufführung von Computermusik oder bei der Musikanalyse als Datenanalyse - damit meine ich sowohl die Analyse von Musik in diagrammatisch-notierter als auch in klanglich-gespeicherter Form - auf eher oberflächlicher

\footnotetext{
1 Felix Stalder, Kultur der Digitalität, Berlin 2016.

2 Vgl. dazu w. u., Fn. 9.
} 
Basis zusammen. Ihr Ensemble und ihre Verknüpfung sind noch viel tiefgreifender. Die Musik spielt sozusagen stets im Computer, noch bevor er ausdrücklich für die Synthese oder Analyse von Klängen programmiert wurde. Dass der Computer also stets eine Musikmaschine ist, kommt im von mir formulierten Neologismus Algorhythmus ${ }^{3}$ zum Ausdruck. Hier habe ich das Wort Algorithmus, wie wir es kennen, mit dem Wort Rhythmus synthetisiert. Abstraktsymbolische Technomathematik in Form gespeicherter Algorithmen zeitigt sich, wenn sie in Laufzeit übergeht, als Algorhythmus real existierender elektronischer Signale. Durch die kultur- und medienwissenschaftliche Aufarbeitung einer Hörkultur, die ich als algorhythmische Hörpraxis bezeichnete, zeigte ich, dass diese ungefähr auf den Zeitraum zwischen 1949 und 1962 abgrenzbare, aber fast global praktizierte Ingenieurpraxis Rechenprozesse durch einfache Verstärker-Lautsprecher-Schaltungen verklanglichte. Signale in der Elektronik der Großrechner wurden abgenommen, elektroakustisch verstärkt und an Lautsprecher geschickt. Dadurch wurden zum Beispiel Operationen wie die Zwischenspeicherung, die jeder Algorithmus als Infrastruktur benutzt, und die sich im Rechner als elektronische Signale manifestieren, durch einen Lautsprecher, der sie in Schalldruckwellen umwandelt, hörbar gemacht. ${ }^{4}$ Algorithmen wurden hier wortwörtlich zu Algorhythmen. Diese einfachen Klangsysteme waren die ersten Schnittstellen und auditiven Interfaces, die computerisierte Rechenprozesse in Echtzeit ästhetisieren konnten und damit sinnlich erfassbar machten. Die algorhythmische Hörpraxis war demnach keine künstlerisch-intentionale Praxis der Computermusik, wie sie etwas später in verschiedenen Kontexten wie in den Bell Labs oder in England betrieben wurde, ${ }^{5}$ sondern entstand aus dem praktischen Problem, dass Elektronik verglichen mit Elektromechanik geräuschlos operiert. Dieses Problem ist nicht nur eines, das etwa mit der Wissens- und Mediengeschichte des Lautsprechers und der medizinischen Praxis der Auskultation ${ }^{6}$ zusammenhängt und den Lautsprecher als Wandler zwischen Klang und Elektronik, das heißt zwischen der Dynamik der Luftmoleküle und jener der Elektronen, einsetzt, sondern dieses Problem entfaltet auch ein pädagogisches Potential: Der Lautsprecher kommt als pädagogisches Spielzeug und -prinzip zum Einsatz, um unhörbare Prozesse des Digitalen hörbar zu machen.

\section{Pädagogische Übungen}

Während Lautsprecher elektronische Signale in akustische Wellen entsprechend ihrem sogenannten Frequenzgang umwandeln, sind neuere, digitale Prozesse oft um ein Vielfaches zu schnell für unsere Ohren. Die Mikroschritte eines Algorithmus, der in modernen Halbleiter-

3 Shintaro Miyazaki, Algorhythmisiert. Eine Medienarchäologie digitaler Signale und (un)erhörter Zeiteffekte, Berlin 2013.

4 Vgl. dazu ebd., S. 78-101.

5 Ebd., S. $213 \mathrm{ff}$.

6 Axel Volmar, "Stethoskop und Telefon. Akustemische Technologien des 19. Jahrhunderts”, in: Das geschulte Ohr. Eine Kulturgeschichte der Sonifikation, hrsg. von Andi Schoon und Axel Volmar, Bielefeld 2012, S. 71-93. 
Computerchips wirkt, zeitigen sich heutzutage zu schnell um sie einzeln zu hören. Trotzdem lassen sich viele gröbere Rhythmisierungen und langsamere, organisatorische Prozesse der Computerhardware digitaler Geräte wie Laptop, Tablet oder Smartphone durch das Anbringen einer Spule oder eines sehr langen Kabels und einem hohen Maß an Verstärkung hörbar machen. Auf ähnliche Weise werden auch elektromagnetische Felder als Störgeräusch in Audiosystemen hörbar. Darüber hinaus existieren Ansätze, die Prozesse im Computer als Algorithmen durch einfache Sonifikation zu verklanglichen.

Um dies zumindest ansatzweise zu erproben, habe ich sogenannte Sortierungs-Algorithmen im Rahmen der Programmiersprache Python sonifiziert. Die Sortierung einer ungeordneten Zahlenreihe in aufsteigender oder absteigender Ordnung ist ein einfaches Programmierproblem, das in der Informatiker*innenausbildung sehr früh erlernt wird. Man kann damit etwas über die Effizienzsteigerung von rekursiven Verfahren und anderen algorithmischen Techniken erlernen und erproben. Mit Hilfe des Kommunikationsprotokolls OpenSoundControl, womit ein Synthesizer der Software Supercollider gesteuert werden kann, habe ich Zahlenwerte, die mir für die Operativität eines Sortierungsalgorithmus wichtig erschienen, als Tonhöhe verklanglicht. ${ }^{7}$ Die erfahrene Leserin mag den sogenannten Print-Befehl kennen, der in vielen Programmiersprachen existiert, um im Programm gespeicherte Werte, wie jene, die in einer If-then-Schleife operativ sind, durch das Anbringen bzw. Notieren in die entsprechende Programmzeile als Zeichenkette an eine sogenannte Konsole auszugeben - ein wichtiger Befehl, um ein Programm auf Fehler zu überprüfen. In meinem Programmierexperiment wurden diese Werte nicht wie üblich in Alphanumerik visualisiert, sondern als Tonhöhe verklanglicht. Mit diesem kleinen Trick, der in seinem epistemischen Gestus dem oben beschriebenen Eingriff in Großrechner mittels eines Verstärker-Lautsprechers stark nahekommt, lassen sich Rhythmen und Frequenzen, viele Routinen, Programmschleifen und andere Operationen hörbar machen. Der Lautsprecher dient hier als pädagogisches Spielprinzip, nicht Spielzeug. Anders formuliert lässt sich das Prinzip mit dem Anbringen von Metallglocken an die Türklinke vergleichen. Jedes Mal, wenn jemand diesen betätigt, wird dies hörbar.

Auf ähnliche Weise operiert die Sonifikation von Algorithmen. Der Print-Befehl wird durch einen virtuellen Lautsprecher, der die Werte in Frequenzen umwandelt, ersetzt. Mit ein wenig Übung, Geschick und Erfahrung lassen sich damit Zeitstrukturen und Operativitäten mancher Algorithmen als Algorhythmus erfahrbar machen. Dies erlaubt eine Wissensproduktion, die nicht nur sprachlich-diskursiv oder visuell-imaginativ, sondern auch rhythmisch und klanglich erfolgen kann. Weitere pädagogisch-didaktische Experimente mit Studierenden der Medienwissenschaft, die das Potential dieses Ansatzes erproben und ausloten, sind derzeit in Planung. Die Programmierumgebung EarSketch, initiiert von einer Forschergruppe am Georgia Institute of Technology, ${ }^{8}$ verfolgt einen ähnlichen, jedoch stärker musikorientierten Ansatz. EarSketch ist

7 Siehe dazu meine Videodokumentation, <https://vimeo.com/334392447> (20.07.2020).

8 <http://earsketch.gatech.edu/> (20.07.2020). 
eine Lernumgebung, die Programmieren mit sampling-basierter Musikproduktion kombiniert und ist zugleich eine computerbasierte Re-Mix-Umgebung für das Lernen einführender Computing-Konzepte. Mit EarSketch lässt sich die Verzeitlichung wichtiger Rechenoperationen wie Modulo als rhythmische Taktverschiebung hörbar machen. Die Lernumgebung ist vielversprechend, weil sie auch für das kollaborative Programmieren in Echtzeit eingesetzt werden kann. EarSketch zeigt ebenso die Nähe zwischen musikalischen und algorithmischen Prozessen, die vor allem in Bezug auf ihre Ähnlichkeit im Zeitlich-Rhythmischen besteht.

\section{Rhythmus als Brückenbilder}

Ein bisher relativ unbekannter Ansatz einer erweiterten kritischen Theorie, die Anknüpfungspunkte zur Musikwissenschaft bieten könnte, ist die sogenannte Rhythmusanalyse, die durch den marxistischen Philosophen und Soziologen Henri Lefebvre und seine postum 1992 erschienene Monographie Rhythmusanalyse geprägt wurde. ${ }^{9}$ Eine Rhythmusanalyst*in hört nicht nur, wie die Psychoanalystin, auf die semantischen Äußerungen eines Menschen, sondern ihre Ohren gelten den Rhythmen von allerlei Geräuschen der Stadt, der Landschaft, deren Menschen, Maschinen und Tiere etc. Dabei wirkt der Rhythmus transversal und konzipiert eine strukturelle Verwandtschaft außermusikalischer, sozialer, affektiver, sozio-psychologischer, politisch-ökonomischer mit musikalischen Prozessen. Die Analyse von Rhythmen scheint hier vielversprechend, da sie laut Lefebvre quantitative und qualitative Aspekte verschränkt.

Verglichen mit den Rhythmen, die ich oben erwähnte, spielen jene, die Lefebvre meint, eher nicht im Bereich von Sekunden, sondern eher von Minuten, Stunden oder gar Tagen. Obwohl solche Rhythmen selbstverständlich unhörbar sind, gibt es seit jeher ebenso Versuche, auch die Stunden und Tage in Musik zu transformieren. Zeitproportionen und -struktur wurden beibehalten und so schnell rhythmisiert, dass sie dann im hörbaren oder musikalisch rezipierbaren Zeitbereich operierten. Geht es so verstanden darum, gesellschaftliche Zeitigungen beobachtbar und adressierbar zu machen, dann bestehen stets auch Möglichkeiten ihrer Verklanglichung und Rhythmisierung. Ob solche zusätzliche Ästhetisierungen, die nicht auf dem konventionellen, aber auch gut erprobten und eingelernten Bildwissen beruhen, sondern ein Hörwissen fordern, neue oder wertvolle wissenschaftliche Erkenntnisse generieren, ist eine Frage, die ich hier außen vor lasse. Auf jeden Fall bietet sich eine Erweiterung eher konventioneller, ästhetischer Erkenntnisformen, die vorwiegend auf das Visuelle setzen, an, zumal diese ein produktives, pädagogisches Potenzial aufweisen könnten.

Digitale Technologien sind seit mehr als zehn Jahren nicht nur allgegenwärtig und dringen in alle Bereiche der Gesellschaft ein. Sie operieren zudem oft unsichtbar etwa in kabelloser, elektromagnetischer Form. Es könnte sich als pädagogisch wertvoll erweisen, so mein Vor-

9 Henri Lefebvre, Élements de rythmanalyse, Paris 1992; hier zitiert nach der englischen Übersetzung: Rhythmanalysis: Space, Time and Everyday Life, London und New York 2004. 
schlag, wenn die vielfältigen Aktivitäten dieser Technologien, das heißt ihre Algorhythmen von der Mikro- bis zu Makro- und Metaebenen der politischen Ökonomien unserer Biotechnosphäre hörbar gemacht werden könnten. Algorhythmen zeitigen sich als seltsame, rekursive Verschränkungen von Technomathematik, Soziomaterialität, Machtstrukturen und -interessen, die im Dazwischen maschinell-digitaler und umweltlich-bio-physikalischer Räume, Kanäle, Netzwerke oszillieren. Ähnlich wie im europäischen Mittelalter die Welt mit Hilfe europäisch-tonaler Musik und Harmonik, insbesondere ihrer ganzzahligen Verhältnisse, erklärt wurde, so könnte die aktuell herrschende Musik des neoliberalen Spätkapitalismus mittels der oben erwähnten Rhythmusanalyse als Algorhythmusanalyse erweitert, analysiert und situiert werden. Hier könnte es sich als produktiv erweisen, wenn wir nicht nur den Rhythmus als epistemisch-ästhetisches Denkwerkzeug betrachten, sondern auch die ganze Palette des musiktheoretischen und klangästhetischen Jargons zu Hand nehmen und auf ihr erkenntniskritisches und pädagogisches Potential überprüfen. In Frage kämen etwa: Signal, Tonalität, Timbre, Harmonie, Kontrapunkt, Dynamik, Artikulation, Dämpfung, Filterung, Verstärkung, Modulation, Transposition, Intervall, Iteration, Synthese, Oszillation, Synchronisation und vieles mehr.

Am Horizont des skizzierten Ansatzes steht schließlich eine kritische Pädagogik der Digitalität, die die Begriffe solch einer vorläufigen Liste aus der Mitte des Interferenzfeldes, wo sich die Musik-, Klang-, Audio-, Medien- und Computerwissenschaften begegnen, re-interpretiert, anwendet und umdeutet, so dass dann diejenigen, die es für notwendig erachten, sich aus einer unerwünschten, ungerechten sozio-medientechnologischen Situation befreien oder vielmehr sie verändern könnten. Weil ein Horizont nie erreichbar ist, bleibt er im besten Fall eine Navigationshilfe der Gegenwart. Ich hoffe, dass dieser kurze Beitrag Ähnliches zu leisten vermag.

Zitation: Shintaro Miyazaki, „Musik für Maschinen?! - Wo sich die Wissenschaft der Medien, des Computers und der Musik treffen und wie sie zusammenarbeiten könnten", in: Brückenschläge zwischen Musikwissenschaft und Informatik. Theoretische und praktische Aspekte der Kooperation, in Verbindung mit der Fachgruppe Digitale Musikwissenschaft hrsg. von Stefanie Acquavella-Rauch, Andreas Münzmay und Joachim Veit (= Musikwissenschaft: Aktuelle Perspektiven. Bericht über die Jahrestagung der Gesellschaft für Musikforschung 2019 in Paderborn und Detmold, Bd. 3), Detmold, Musikwissenschaftliches Seminar der Universität Paderborn und der Hochschule für Musik Detmold, 2020, S. 173-178, DOI: 10.25366/2020.105 


\section{Abstract}

Musicology and computer science do not only come together in areas such as the use, analysis and performance of digitized data, but also meet in unexpected places such as in context of critical media studies and inquiries about the material and aesthetic conditions of digitality. Such "exploratory interactions" with computers and their aesthetics might resonate well with musicology. This mini-contribution firstly presents a historical situation in which for a short period in the 20th century the machinic music of digitality became audible. It then formulates, just as briefly and sketchily, the socio-critical potential of music-oriented approaches, especially rhythm analysis, still to be tested, when it comes to grasp and understand digitality in as many facets as possible (socio-technological, aesthetic, historical and epistemic). Thereby, a fourth partner might be of importance: pedagogy.

\section{Kurzvita}

Shintaro Miyazaki, geboren 1980, studierte Medienwissenschaft, Philosophie und Musikwissenschaft an der Universität Basel (2000-2007). 2012 promovierte er in Medientheorie und -wissenschaft an der Humboldt-Universität zu Berlin. 2014-2020 war er Wiss. Mitarbeiter und Senior Researcher an der Hochschule für Gestaltung und Kunst der Fachhochschule Nordwestschweiz in Basel. Seit Oktober 2020 ist er Juniorprofessor für "digitale Medien und Computation" im Fachbereich Medienwissenschaft der Humboldt-Universität zu Berlin. 



\section{Brückenschläge zwischen Musikwissenschaft und Informatik}

Theoretische und praktische Aspekte der Kooperation

Herausgegeben von Stefanie Acquavella-Rauch,

Andreas Münzmay und Joachim Veit 
Brückenschläge zwischen Musikwissenschaft und Informatik 


\section{Musikwissenschaft: Aktuelle Perspektiven}

Bericht über die Jahrestagung der Gesellschaft für Musikforschung 2019 in Paderborn und Detmold

Herausgegeben von Rebecca Grotjahn und Nina Jaeschke

Band 3 


\section{Brückenschläge zwischen Musikwissenschaft und Informatik}

Theoretische und praktische Aspekte der Kooperation

Beiträge der Symposien zur Digitalen Musikwissenschaft

Osnabrück 2018 und Paderborn 2019

im Rahmen der Jahrestagungen der Gesellschaft für Musikforschung

In Verbindung mit der Fachgruppe Digitale Musikwissenschaft herausgegeben von

Stefanie Acquavella-Rauch, Andreas Münzmay und Joachim Veit

Detmold: Musikwissenschaftliches Seminar der Universität Paderborn und der Hochschule für Musik Detmold 2020 
DOI: $10.25366 / 2020.87$

Online-Version verfügbar unter der Lizenz: Urheberrecht 1.0, $<$ https://rightsstatements.org/page/InC/1.0/?language =de >

Bibliografische Information der Deutschen Nationalbibliothek

Die Deutsche Nationalbibliothek verzeichnet diese Publikation in der Deutschen Nationalbibliografie; detaillierte bibliografische Daten sind im Internet über http://dnb.d-nb.de abrufbar.

\section{Impressum}

Redaktion: Stefanie Acquavella-Rauch, Andreas Münzmay und Joachim Veit Satz: Nina Jaeschke und Joachim Veit

(C) Musikwissenschaftliches Seminar der Universität Paderborn und der Hochschule für Musik Detmold 2020 


\section{INHALT}

Rebecca Grotjahn, Nina Jaeschke

Vorwort zu Band 1-3

IX

Stefanie Acquavella-Rauch, Andreas Münzmay, Joachim Veit

Brückenschläge zwischen Musikwissenschaft und Informatik - Vorbemerkung

$\mathrm{XI}$

\section{KOLLABORATIONEN - KO-LABORATORIEN}

\section{Reinhard Keil}

Der Computer als Denkzeug für hermeneutische Arbeit

\section{Ulrich Konrad}

Philologie und Digitalität. Perspektiven für die Musikwissenschaft im Kontext fächerübergreifender Institutionen

\section{Gudrun Oevel}

Infrastruktureinrichtungen in Forschungsprojekten - Spagat oder Chance?

\section{Dennis Ried}

Erhebung, Transformation und Präsentation digitaler Forschungsdaten

Anna Neovesky, Frederic von Vlahovits

IncipitSearch - Leitfaden zur Zusammenarbeit

Elisabeth Treydte

Clara Schumann \#digital. 40 Jahre Archiv Frau und Musik und der Start in die Digitalisierung

\section{TEXT/DATEN/PROZESSE}

\section{Christine Siegert}

Komponisten-Gesamtausgaben im digitalen Zeitalter: Perspektiven

und Reflexionen am Beispiel Ludwig van Beethovens

Markus Neuwirth, Johannes Hentschel, Martin Rohrmeier

Perspectives of Musical Corpus Studies: The Annotated Mozart Sonatas

Agnes Amminger, Franz Kelnreiter

Leopold Mozarts „Gründliche Violinschule". Zur Textcodierung und -präsentation einer digitalen Edition

\section{Oleksii Sapov}

Algorithmische Automatisierung komplexer Notationsregeln in MEI-XML am Beispiel von Versetzungszeichen 


\section{Susanne Cox, Richard Sänger}

Digitale Fassungsvergleiche am Beispiel von Beethovens Eigenbearbeitungen

Agnes Seipelt

Digitale Edition und Harmonische Analyse mit MEI von Anton Bruckners

Studienbuch

Stefanie Acquavella-Rauch

Musikalische Schaffensprozesse 2.0 - Inkorporation audiovisueller Medien

der populären Musik in Methoden der digitalen Edition

\section{DIGITAL(ISIERT)E MATERIALITÄTEN}

\section{Miriam Akkermann}

(Musik)Instrument (im) Computer

\section{Daniel Fütterer}

Herausforderungen bei der Kodierung von Paratext am Beispiel Neuer Musik mit Live-Elektronik

\section{Matthias Pasdzierny}

How much is the glitch? Das digitale Paradigma als Herausforderung

und Chance für die historische Musikwissenschaft

\section{Shintaro Miyazaki}

Musik für Maschinen?! - Wo sich die Wissenschaft der Medien, des Computers und der Musik treffen und wie sie zusammenarbeiten könnten

\section{MUSIKGESCHICHTE(N) IM NETZ}

\section{Matthias Tischer}

Musikgeschichte der DDR: Ein Pilotprojekt zur digitalen Musikvermittlung

Annette van Dyck-Hemming, Jan Eberhardt, Melanie Wald-Fuhrmann

Ansätze zur Analyse historischer Netzwerke mit Neo4j® - Aus der Projekt-Werkstatt der Datenbank zur Fachgeschichte der Musikwissenschaft

Axel Beer, Martin Bierwisch, Kristina Krämer

Das MMM2 - Ein regionalgeschichtliches Onlinelexikon der Arbeitsgemeinschaft für mittelrheinische Musikgeschichte

\section{Matej Santi}

Was erzählt Fritz Kreislers Geige?

\section{Elias Berner}

Alle Menschen werden Brüder?! Ein historisches Dokument aus dem

Nationalsozialismus in den sozialen Medien 
Gabriele Buschmeier in memoriam 